\title{
TOWARDS UNDERSTANDING THE
}

\section{ANGIOMOTIN MEMBRANE FUSION}

\section{ACTIVITY}

\author{
Seth Sears, Brenner Evans and Ann C. Kimble-Hill*
}

Dept. of Biochemistry \& Molecular Biology, Indiana University School of Medicine, MS4053, 635 Barnhill Dr., Indianapolis, IN 46202, USA.

*denotes corresponding author.

ABSTRACT: Angiomotins (Amot) are a family of adaptor proteins that regulate cellular differentiation and proliferation signaling. These cellular events are linked to the initiation of invasive ductal carcinoma, the most common form of breast cancer. Their characteristic coiledcoil homology $(\mathrm{ACCH})$ domain selectively binds phosphatidylinositol lipids (PI), leading to membrane deformation and fusion events necessary for the trafficking polarity proteins to and from the apical membrane. We have previously shown that the ACCH domain has the ability to reorganize and homogenize the microdomains driven by the presence of PIs in membranes. However, the connection between the ability of this domain to facilitate membrane fusion and to homogenize PI driven lipid phases has not been well described. In this study, we use small angle x-ray scattering (SAXS) to demonstrate the lipid reorganization function of the ACCH domain while using fluorescence resonance energy transfer (FRET) to characterize the vesicle fusion 
activity. As a result, we found 6 mutations in the ACCH domain that caused a significant or total loss of fusion activity. Furthermore, mutations that retard this membrane fusion activity also have lost the ability to homogenize the PI lipids within the membrane. The results presented provide insight into the role of the Amot $\mathrm{ACCH}$ domain residues in driving membrane fusion events involved in targeting and recycling cellular polarity proteins to maintain normal cellular phenotypes.

KEYWORDS. Angiomotin, phosphatidylinositol, membrane fusion, lipid microdomains.

\section{Introduction}

The family of Angiomotin (Amot) proteins regulate several biological pathways associated with cellular differentiation, proliferation, and migration. The Amot adaptor protein integrates the control of cellular morphology with its tendency to proliferate.[1-3] This is mainly accomplished through the differential ability of specific isoforms of Amot to orient the trafficking of proteins that regulate apical cell polarity and related growth control pathways.[3-12] Amot membrane association is due to the Amot coiled-coil homology domain (ACCH),[13] a predicted coiled coil fold of approximately 240 residues that has the unique property of being able to selectively bind phosphatidylinositols (PI) and cholesterol as well as fuse golgi vesicles enriched in 4'phosphorylated PIs to the plasma membrane and induce evagination of the plasma membrane to recycle proteins in early stage endosomes enriched in 3'phosphorylated PIs.[13] Such associated binding events lead to vesicle fusion at the cellular apical membrane. This activity is thought to be a result of the binding and reshaping of vesicles saturated with these mono-phosphorylated PI. Overexpression of Amot, and therefore its related activity, has been correlated with abnormal cell mortality, cell growth and proliferation due to the cells inability to recycle the polarity proteins to 
and from the plasma membrane.[14] Previous reports suggest that the ACCH domain carries out this membrane deformation through a similar manner to that of BAR domains[13] and fusion activity like coiled coil domain of NUDEL or SNAREs[15] where the N-terminus serves to drive oligomerization and the C-terminus drives membrane fusion. Therefore, we have endeavored to further understand this mechanism using biophysical techniques.

We previously used fluorescence quenching experiments to demonstrate nonphosphorylated PI induction of co-existing lipid phases within ternary mixtures with phosphocholine and phosphoethanolamine, where interaction with the ACCH domain causes the lipids to become reorganized into a more homogenous system.[16] Hence, we hypothesized that the membrane fusion activity was dependent on the ability of the ACCH domain to reorganize and homogenize the lipids. In this work, we use small angle x-ray scattering (SAXS) to gain insight into the mechanism behind this activity. As a result, we were able to use SAXS measurements to demonstrate the $\mathrm{ACCH}$ domain reorganization function of the microdomains in this PI lipid mixture into a more homogeneous membrane. Then using Fluorescence Resonance Energy Transfer (FRET), we determined that the mutation of specific arginine and lysine residues within the ACCH domain led to a reduction in the protein concentration dependence on vesicle fusion (Lys-72, Lys-187, Arg-234), while other mutations were able to severely retard or obliterate this function (Arg-40, Lys-49, Lys76, Arg-103, Lys-111, Lys-136, Arg-221, and Arg-224). Finally, we used SAXS to show that the Lys-49 ACCH mutant also lacked the ability to reorganize PIcontaining membranes. Therefore, we were suggest that $\mathrm{ACCH}$ domain membrane fusion is necessary for measurable lipid reorganization activity to occur.

\section{Results \& Discussion}


ACCH Domain Reorganizes Lipid Phases. Figure 1A shows small angle X-ray scattering (SAXS) data collected on multi-lamellar vesicles composed of POPC/POPE/PI (60/20/20 mol\%) under shear stress from a microfluidics device. This system contained four dominant Bragg peaks at $\sim 0.104,0.116,0.119$, and $0.122 \AA-1$ along with several other peaks of lesser intensity. The most dominant of these peaks, 0.119 and $0.122 \AA$-1, have significant changes in intensity are observed as the ACCH domain concentration increases in the solution (Figure 1B). Specifically, the 0.1160.122 peaks appear to form a merged peak at $\sim 0.122 \AA$-1 with a shoulder at $\sim 0.126 \AA$-1.

The four dominant peaks of this lipid mixture are a part of a series of reflections that can be fitted to 4 lamellar co-existing space groups that have not been previously reported for this mixture (Figure 1C).[16] The first lamellar space group contained Bragg peaks at q (I/Imax) 0.052 (0.012), 0.104 (0.020), 0.155 (0.012), 0.205 (0.010), 0.260 (0.009), and $0.312(0.009) \AA$ A 1. The second lamellar space group contained Bragg peaks at q (I/Imax) $0.060(0.015), 0.119$ (0.044), $0.181(0.011), 0.241(0.009)$, and $0.300(0.009) \AA$-1. The third lamellar space group present contained Bragg peaks at $\mathrm{q}(\mathrm{I} / \mathrm{Imax}) \sim 0.063$ (0.015), $0.122(0.016), 0.189$ (0.011), $0.252(0.009)$, and 0.314 (0.009) $\AA$-1. The final lamellar space group associated with these peaks of interest contained Bragg peaks at q (I/Imax) 0.116 (0.064), 0.231 (0.009), $0.348(0.009) \AA-1$. The peak positions were indexed and fitted to Equation 2 resulting in lamellar d-spacings of $120.7 \pm 0.7$ ( $R_{2}$ $=0.9998), 104.3 \pm 0.5\left(\mathrm{R}_{2}=0.99992\right), 99 \pm 1\left(\mathrm{R}_{2}=0.9996\right)$, and $54.2 \pm 0.2\left(\mathrm{R}_{2}=0.99998\right) \AA$.

As the lipid are incubated with increasing protein concentrations, the three most dominant peaks become $\sim 0.092,0.103$, and $0.123 \AA$-1. These dominant peaks as a result of incubation with $0.07 \mathrm{mM}$ protein were assigned to 4 lamellar co-existing space groups (Figure 1E). The first lamellar phase contained Bragg peaks at q (I/Imax) 0.045 (0.011), 0.092 (0.014), 0.139 (0.011), 0.180 (0.010), 0.224 (0.008), 0.271 (0.007), 0.314 (0.007), and $0.362(0.007) \AA-1$. The second 
lamellar space group contained Bragg peaks at q (I/Imax) $0.050(0.011), 0.103(0.015), 0.150$ (0.010), 0.200 (0.009), 0.253 (0.008), 0.300 (0.007), $0.351(0.007)$ and $0.392(0.007) \AA$-1. The third lamellar space group present contained Bragg peaks at q (I/Imax) $0.061(0.010), 0.123(0.042)$, $0.181(0.010), 0.244(0.008), 0.307$ (0.007), and $0.366(0.007) \AA$-1. The peak positions were indexed and fitted to Equation 2 resulting in lamellar d-spacings of $140.1 \pm 0.9\left(\mathrm{R}_{2}=0.9998\right), 128$ $\pm 1\left(\mathrm{R}_{2}=0.9994\right)$, and $102.8 \pm 0.6\left(\mathrm{R}_{2}=0.9999\right) \AA$.

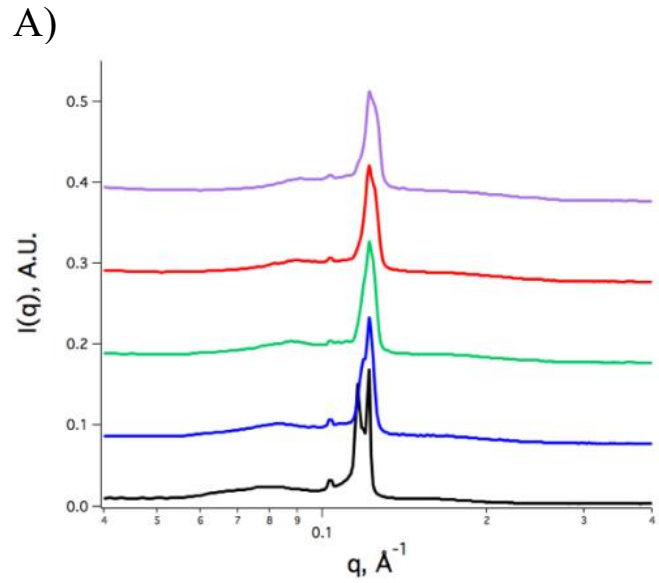

C)

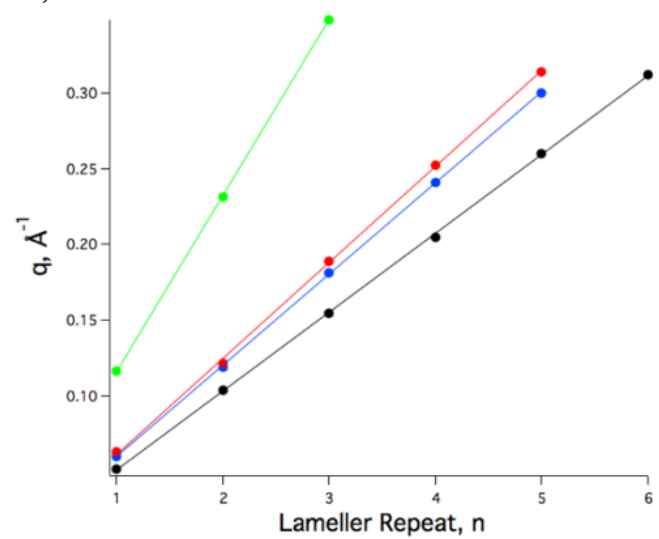

B)

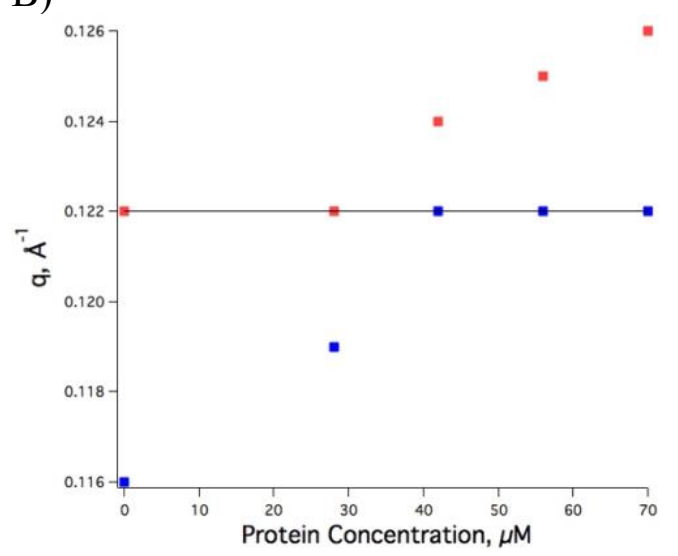

D)

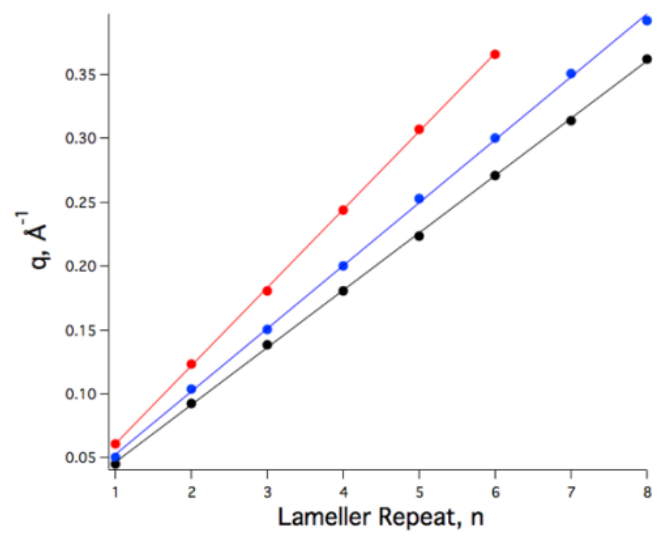

Figure 1. Effect of ACCH Domain on POPC/POPE/PI 60/20/20 mol\% lipid phase arrangements. A) Small angle x-ray scattering patterns of $20 \mathrm{mM}$ lipid incubated with $0,0.028,0.042,0.056$, and $0.07 \mathrm{mM}$ wt protein (-, -, -, -, - respectively). B) Positions of the 2 most dominant Bragg peaks that move as a function of protein concentration, where the doublet 1 st and 2 nd peak position (blue, red respectively). Lamellar Bragg peak repeats present in mixtures C) without protein compared to D) with $0.07 \mathrm{mM}$ protein. 
In comparison to the d-spacings found for the lipids in their native state, the $\mathrm{ACCH}$ domain causes the appearance of a new much larger phase and the loss of the smallest phase. Additionally, it appeared as if the two remaining lamellar space groups were both swollen by the presence of the protein as the $\sim 121 \AA \mathrm{d}$-spacing grew larger by $\sim 7 \AA$ and the $99 \AA$ d-spacing grew larger by $3 \AA$. Increases in d-spacing was expected as the water layer of the lamellar repeats most likely grew larger to accommodate the presence of the protein as they interact with the lipid head groups. Additionally, the presence of multiple lipid phases has been linked to phosphocholine enriched regions of the membrane (without shear stress $\sim 55-85 \AA$ ) [16, 17] co-existing with PI enriched microdomains (without shear stress $\sim 50-69 \AA$ ). [16] The loss of the smallest phase of this mixture as a result of being incubated with the ACCH domain suggests that the PI is predominantly found in the $\sim 54 \AA$ phase. It also further demonstrates that interactions of this phase with the ACCH domain decreased the PI preference to form co-existing phases and increased preference to form more homogenous membranes. Furthermore, the peak previously seen at $0.116 \AA-1$ before incubation with the protein was consumed within the shoulder of the new $0.122 \AA-1$ peak, along with the appearance of a new shoulder peak at $0.126 \AA$-1. This is further indication that the lipid phases have been reorganized as a result of interaction with the ACCH domain. This result aligns with our previous fluorescence quenching studies showing that the ACCH domain decreases the appearance of co-existing phases.[16] This result would suggest that Amot association with either golgi apparatus vesicles or early endosomal vesicles would cause a reorganization of the PI lipids thereby creating more homogenous vesicular membrane targeted for downstream events. However, the literature does not suggest a specific role for this lipid reorganization in the same way that Amot activity has been linked to maintaining normal polarity protein activity. Heller et al (2010) used cro-electron micrographs and dynamic light scattering to show that the ACCH 
domain causes liposomes to merge into much larger structures.[13] This in vitro activity was linked to the in vivo fluorescent imaging that showed a loss of Amot ACCH domain activity led to a loss polarity proteins being brought to the plasma membrane of epithelial cells. Therefore, we endeavored to understand whether the $\mathrm{ACCH}$ domain membrane fusion activity was linked to its ability to reorganize the phase of the lipids into a more homogenous mixture.

Optimizing a membrane fusion assay. Figure 2 pictorially describes the FRET assay used throughout this work to study the ability of the ACCH domain to fuse liposomes. Here we use a previously described homology structure of the ACCH domain[15] to suggest a model for the fusing of liposomes undergoing a similar mechanism as SNARE proteins.[15]

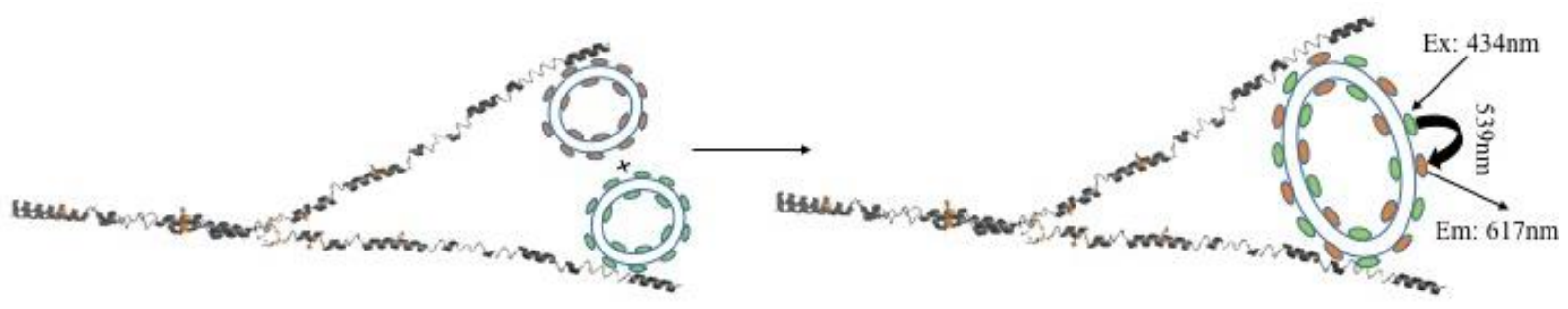

Figure 2. FRET Assay Model of ACCH domain mediated fusion of POPC/POPE/POPI vesicles.

We characterized the time dependence of the ACCH domain fusion activity and found that 3/1/1 molar ratio POPC/POPE/PI liposomes exhibited vesicle fusion after nine hours where the fusion plateaued after 14 hours (data unshown). Using this same assay, we were unable to detect any measurable vesicle fusion within a 24-hour period in 3/1/1 molar ratio POPC/POPE/POPA, a lipid mixture previously reported as not undergoing $\mathrm{ACCH}$ domain related fusion.[13] We then endeavored to use mutants from our library that did not have this membrane fusion, to determine whether membrane fusion preceded lipid reorganization. The arginine and lysine mutant library was originally designed to screen for loss of membrane organization and has been described 
elsewhere.[18] Mutations that led to a decrease in protein association with PI containing membranes were not used in this study. We decided to screen the resulting mutants for a loss of ability to cause vesicle fusion.

Screening Mutants for Loss of Fusion Activity. We then used this assay to screen mutants for decreased vesicle fusion after a 16-hour incubation. For comparison, we included the $\% \mathrm{Max}$ FRET for the wild type protein in the presence of 3/1/1 POPC/POPE/POPA (NC) and 3/1/1 POPC/POPE/PI (PC). As a result, we found 11 mutants that led to a $>60 \%$ reduction in fusion: R40T, K49E, K72E, K76E, R103G, K111E, K136E, K187E, R221Q, R224E, R234G. Figure 3 shows the predicted secondary structure model of the ACCH domain.[13]

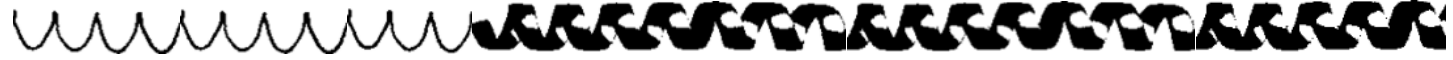 MPRAQPSSASYQPVPADPFAIVSRAQQMVEILSDENRNLRQELEGCYEKVARLQ

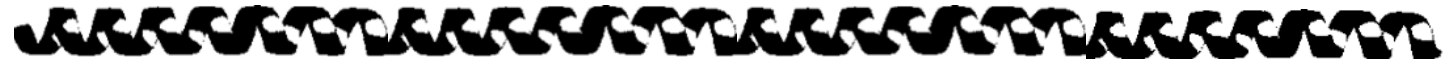 KVETEIQRVSEAYENLVKSSSKREALEKAMRNKLEGEIRRMHDFNRDLRERLE}

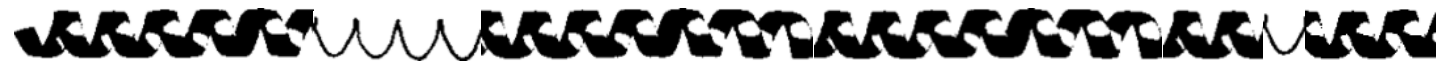
TANKQLAEKEYEGSEDTRKTISQLFAKNKESQREKEKLEAELATARSTNEDQR

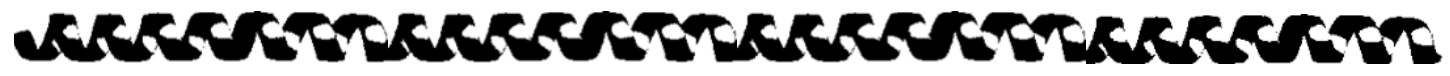
RHIEIRDQALSNAQAKVVKLEEELKKKQVYVDKVEKMQQALVQLQAACEKR

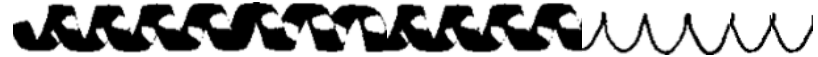

\section{EQLEHRLRTRLERELESLRIQQROGNCOPTNVS}

Figure 3. Predicted secondary structure of ACCH Domain using GOR4 where $85.6 \%$ is $\alpha$-helical, $1.6 \%$ is an extended strand, and $12.7 \%$ is randomly coiled. The residues of interest are highlighted categorically. I) Reduced fusion: K72E (•), K187E (•), R234G (•); II) Higher concentration onset: K49E (•), R103G (•); and III) Obliterated fusion: R40T (•), K76E (•), K111E (•), K136E $(\bullet), \mathrm{R} 221 \mathrm{Q}(\bullet), \mathrm{R} 224 \mathrm{E}(\bullet)$.

The residues under investigation are highlighted categorically based on our suggested mutant classification. Further, Class 1 mutants (green) represent mutants that showed reduced 
lipid-liposomal fusion relative to the positive control. Class 2 mutants (blue) constitute mutations that showed vesicle fusion activity following the burst phase of $0-100 \mathrm{nM}$ protein concentration. Lastly, Class 3 mutants (red) are particularly interesting due to their characteristic ability to completely obliterate vesicle fusion events relative to the positive control.

Characterization of Mutant-Liposome Fusion Activity. After identifying the mutants that caused a significant decrease in vesicle fusion activity, we then determined the protein concentration effect on the ability to cause vesicle fusion. This study illustrated which mutants displayed a reduction in vesicle fusion activity and a complete loss of fusion activity. To address this question, the amount of FRET was measured for each protein concentration after the 16-hour incubation period. Figure 4 shows the results from our ACCH domain driven liposome fusion experiment where the positive control represents the fusion of POPC/POPE/POPI versus the fusion of the negative control POPC/POPE/POPA lipid mixtures. For each mutant, we determined the amount of fusion based on FRET intensity versus protein concentration and reported that slope. As a result, we found that there were three classes of mutations: Class I reduced activity, Class II higher concentration onset, and Class III obliterated activity.

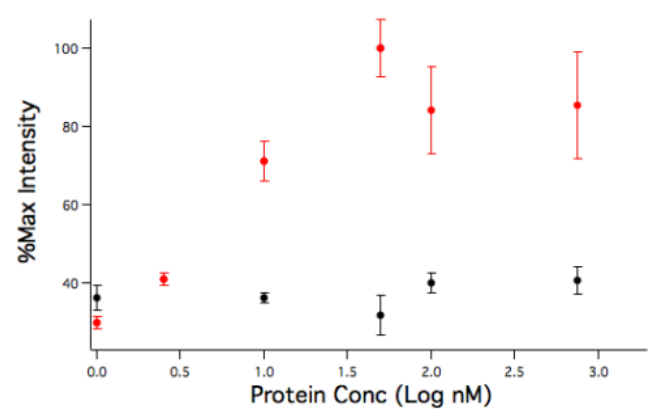

Figure 4. Vesicle Fusion as a function of ACCH domain concentration. FRET between 2 liposome populations either containing a 3/1/1 molar ratio of POPC/POPE/PI $(\bullet)$ as the positive control and the negative control POPC/POPE/POPA $(\bullet)[13]$ driven by incubation with the ACCH domain at various concentrations. 
Class I: reduced activity. Mutations in this class still have appreciable fusion as a function of protein concentration in comparison to wild type protein (Figure 5). Table 1 summarizes the mutations in this class (K72E, K187E, and R234G) and their corresponding slopes. These mutants showed reduced fusion activity in the burst phase (0-100nM) with 2-5-fold reduction in activity. Based on the known helical nature of this domain reported by Heller et al.,[13] it is interesting that Lys-72 falls in the middle of the 1 st predicted alpha helix, Lys-187 in the middle of the 2nd predicted helix, and Arg-234 in the C-terminal random coil region (Figure 3). This suggests that these residues probably do not play a role in the initiation of the fusion. However, if the ACCH domain fusion mechanism is similar to the coiled coil domain of NUDEL or SNARE complex fusion events[19] and BAR domain tubulation events,[20, 21] then these mutations probably are not affecting the overall structure of the helices. Instead, their reduction in fusion ability may be more related to a reduction in the ability of the coils to "crawl" along its oligomer interface to bring membranes into closer proximity to one another. Furthermore, Lys187 falls within the middle of a lysine/arginine sequence (KVVKLEEELKKKQVYVDKVEK) that has been moderately predicted to be a nuclear localization sequence.[22] This residue may be key in the ability of Amot130 to perform the previously reported function of shuttling the transcriptional co-activator Yes-associated protein (YAP) to the nucleus,[23] suggesting that after targeting to the nuclear membrane there would be a decrease in Amot ability to transport YAP into the nucleus. 


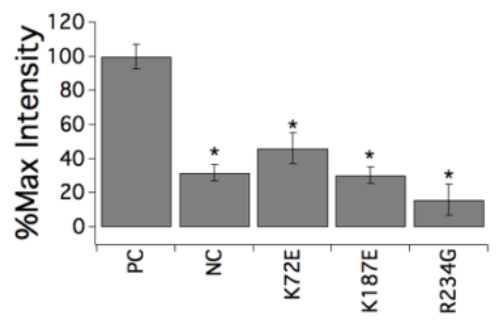

Figure 5. Effect of Class I mutations on ACCH domain membrane fusion activity. Analysis of the statistical significance of each mutant at $50 \mathrm{nM}$ relative to the positive control where $*$ denotes $\mathrm{p}<0.05 * *$ and denotes $\mathrm{p}<0.005$.

Table 1. Summary of Liposome Fusion Rates for Class I: Mutants with Reduced Activity

\begin{tabular}{|c|c|c|c|c|}
\hline Mutation & $\begin{array}{c}\text { Fusion Rate } \\
(\mathrm{nM}-1)\end{array}$ & Std Dev & $\begin{array}{c}\text { Fold } \\
\text { Change }\end{array}$ & $\begin{array}{c}\mathrm{p} \\
\text { Value }\end{array}$ \\
\hline $\mathrm{Wt}$ & 43.0 & 2.0 & - & - \\
\hline $\mathrm{K} 72 \mathrm{E}$ & 19.0 & 5.0 & 2 & 0.02 \\
\hline $\mathrm{K} 187 \mathrm{E}$ & 22.0 & 3.0 & 2 & 0.005 \\
\hline R234G & 10.1 & 2.0 & 4 & 0.006 \\
\hline
\end{tabular}

Class II higher concentration onset. This category of mutants included K49E and R103G.

These mutants showed decreased protein concentration dependence compared to the positive control until around 100nM (log $2 \mathrm{nM})$ protein concentration (Figure 6). At $\sim 100 \mathrm{nM}$ protein concentration, residues $\mathrm{K} 49 \mathrm{E}$ and $\mathrm{R} 103 \mathrm{G}$ begin to show a protein concentration dependence for vesicle fusion activity. 

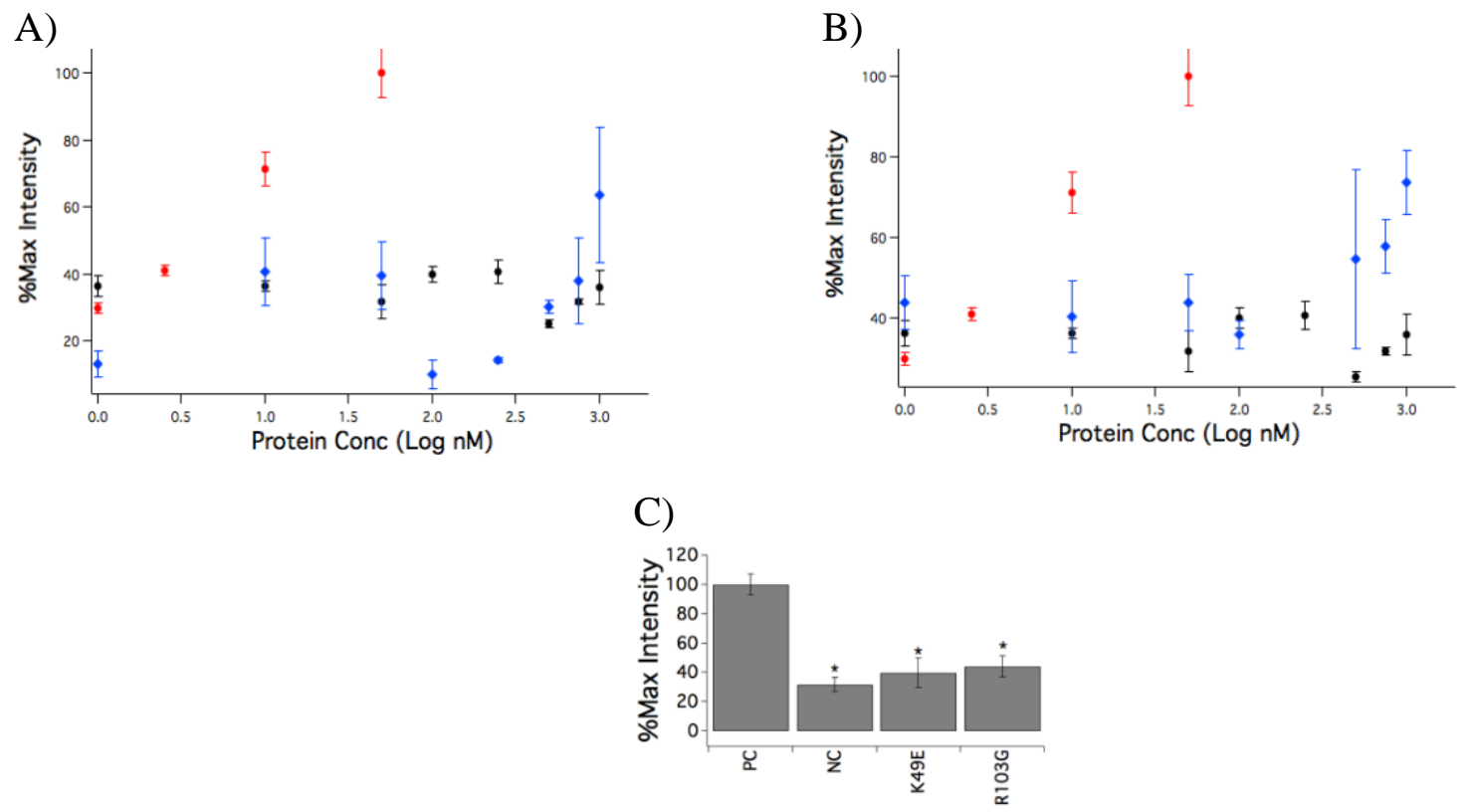

Figure 6. ACCH domains mutations with vesicle fusion at higher concentrations. FRET between 2 liposome populations containing a 3/1/1 molar ratio of POPC/POPE/PI (•) as a positive control or the negative control POPC/POPE/POPA $(\bullet)[13]$ fused as a result of wild type ACCH domain compared against the A) K49E and B) R103G mutations (•). C) Analysis of the statistical significance of each mutant at $50 \mathrm{nM}$ relative to the positive control where * denotes $\mathrm{p}<0.05 * *$ and denotes $\mathrm{p}<0.005$.

Lys-49 is situated in the middle of the 1 st alpha helix and Arg-103 is at the end of the 1 st alpha helix (Figure 3). Interestingly, the late onset of Lys-49 vesicle fusion is a result of changing from a positively charged to a negatively charged residue (Figure 6A). While this mutation is not suggested to affect the ability to form an alpha helix, the much higher concentration and location within the 1 st alpha helix may suggest a reduction in the ability to form dimers in the same manner as other coiled coil domain proteins. More specifically, we suggest that this mutation may cause some repulsion against residues in the other dimer interface thereby reducing the overall ability to fuse vesicles. The mutation of Arg-103 to a glycine also shifted the concentration dependence phase to almost a 100-fold higher concentration range (Figure 6B). This result suggested that the mutation of this residue to the non-bulky, nonpolar 
glycine residue may induce a known ability of glycine to introduce new flexibility into an alpha helical chain thereby affecting the nearby ability to continue the local secondary structure.[24] Additionally, inversion of the alpha helical chain at Arg-103 to glycine likely decreases dimer interface formation with nearby residues. We suggest that this change in structure resulting from glycine flexibility may cause a misalignment of polar and nonpolar residues that participate in the dimerization step similar to that in the SNARE fusion activity.[25] This would ultimately lead to an indirect effect on the protein concentration dependence for vesicle fusion.

Class III: obliterated activity. The final mutant category suggested to have no appreciable fusion activity in this study were R40T, K76E, K111E, R221Q, and R224E. Each of these mutants had no protein concentration dependence on vesicle fusion activity compared to the negative control (Figure 7). The mutation of Arg-40 was found to reduce fluorescent readings to intensities less than or equal to the negative control (Figure 7A). Arg-40 near the beginning of the $1_{\text {st }}$ alpha helix, and our mutation changed this positively charged residue into a threonine, which is a non-charged polar residue. As we already mentioned in the previously studied coiled coil domain proteinss, this 1 st alpha helix is believed to play a major role in dimer, or higher ordered oligomer, formation. When we mutated Lys-49, which is approximately 2.5 alpha helical turns downstream of this mutation, it led to a less drastic effect on vesicle fusion. This would suggest that Arg-40 has a direct role in dimer formation and a mutation of this residue to an uncharged, nonpolar residue obliterated fusion activity.

The fluorescent readings for the concentration dependence of mutating Lys-76 to a glutamate were similar to the negative control (Figure 7B). Similar to Lys-72 (class I), Lys-76 located in the middle of the 1 st alpha helix, may affect the ability of the ACCH domain to oligomerize and fuse membranes. It is interesting that the mutation of Lys-76 to glutamate 
obliterated vesicle fusion activity; however, the mutation of Lys-72 to glutamate only reduced the concentration dependence 2-fold. Being that these residues are 4 residues apart, on the same alpha helix, one could assume that they do not face the exact same dimer interface. Therefore, we suggest that Lys-76 also directly participates in the dimer interface thereby obliterating the ability to form the dimers necessary to have vesicle fusion activity.

Similar to the Lys-76 mutation, the mutations of Lys-111 and Lys-136 to a glutamate also caused a similar concentration dependence as compared to the negative control (Figure 7C and D, respectively). Lysine-111 is located at the end of the 1 st alpha helix outside of the previously indicated "knobs-into-holes" interface while Lysine-136 is found at the $\mathrm{N}$ terminus of the 2nd alpha helix (Figure 3). By introducing these mutations, we observed that amino acid charge reversal has a greater effect on function than the introduction of flexibility at Arg-103. This may indicate that the hinge region of the 1 st alpha helix is important in SNARE-like motif binding. Previous literature reports that mutations in hinge domains that connect $\mathrm{N}$ - and $\mathrm{C}$ - terminal $\alpha$ helical domains of t-SNARE proteins inhibited its essential functional oligomerization of SNARE complexes.[26, 27] While the mechanism by which the ACCH domain binds to membranes is not currently known, a previously proposed model suggests that two ACCH domain dimers associate together and form a tetramer complex that undergoes SNARE-like fusion to membranes.[15] Other reports indicate that the N-terminus of multiple alpha helix bundles may be required for activation of SNARE complexes.[28] By mutating residues Lys-111 or Lys-136 to a negatively charged glutamate, the SNARE-like complexes may be unable to associate together and subsequently unable to cause fusogenic activity.

Coiled coil domains generally have a very specific residue heptad abcdefg pattern where residues $\mathbf{a}$ and $\mathbf{d}$ are hydrophobic.[19, 29] In this dimer interface region of the alpha helix, the 
pattern may have a significant effect on the ability to complete the coiled coil. Our lab previously reported theoretical structures that suggest where these heptads lie within the 1 st alpha helix.[15] Both Lysines 49 and 72 both are participating in the "knobs-into-holes" that form the interconnected tertiary structure. However, Arg-40 (4 residues before the interconnection) and Lys-76 (1 residue after the interconnection) lie directly outside of these localized structures but do fall within the first and third interfaces of the dimer. This suggests that mutations of charge reversal found outside but near these heptad regions play a significant role in stabilizing the overall interface of these dimers. This may also explain the significant decrease in ability of these mutants to fuse the POPC/POPE/PI vesicles compared to the wild type ACCH domain ability to fuse POPC/POPE/POPA vesicles (Figure 7. G).

The proximity of Arg-221 and Arg-224 suggest that both of the positive charges of the side chains are interacting with the negatively charged inositol head groups during fusion activity, which may participate in the direct binding of SNARE-like binding complexes to the membrane as the coils bring the membranes closer to one another during the fusion event. Moreover, residues found in the linker region of the SNARE-like motif are critical for zipper activity to the membrane.[25, 28] Additionally, by mutating Arg-221 and Arg-224 to the opposite charge we suggest that we inhibited SNARE-like zipper activity without affecting the ability of the protein to associate with the membrane. Zippering activity is generally the result of energy released from the SNARE-like complex binding to the membrane. Therefore, by inhibiting the binding complex, the catalytic forces that fuse juxtaposed vesicles to the membrane is diminished.[30] Furthermore, the Arg-221 and Arg-224 mutation effects are unlike the mutation we saw in Arg-234. This residue was suggested to be in the random coil region immediately following this alpha helix,[15] as the c-terminal alpha helix of the coiled coil 
domain proteins generally directly interact with the lipid membrane.[30] Therefore, if the ACCH domain follows in that structure-function relationship we suggest that these mutations would directly affect the ability of full length Amot to fuse endosomes to the apical membrane for polarity proteins recycling and form normal tight junctions.[1-3, 13]

Residues Arg-221 and Arg-224 are suggested to be located near the C-terminus of the 2nd alpha helix (Figure 3). In this study, we mutated Arg-221 to a glutamine, which is a polar, uncharged residue with an almost comparable size side chain but also led to a loss of fusion activity (Figure 7E). The mutation of Arg-224, which is three residues downstream of Arg-221, into a glutamate, introduced a reversal in the native charge, thereby leading to the same effect (Figure 7F).

A)

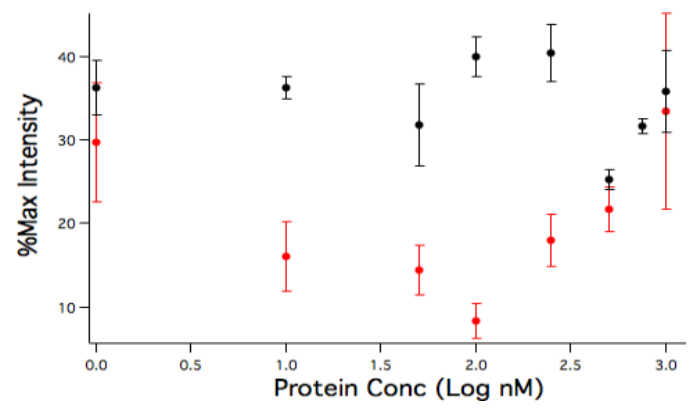

C)

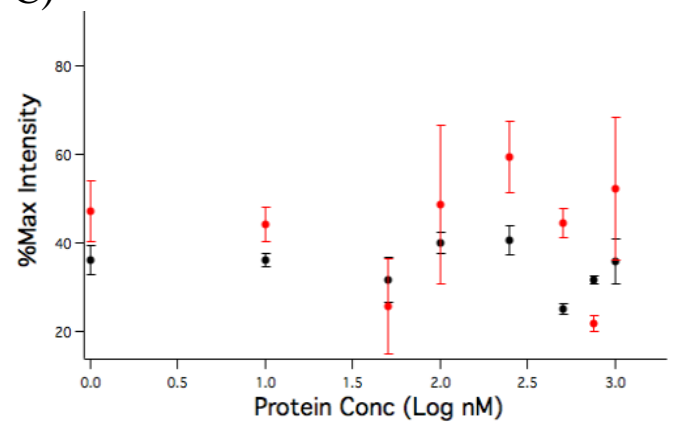

B)

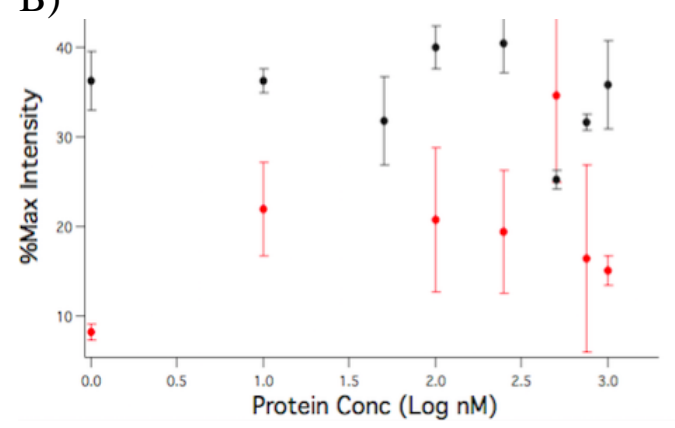

D)

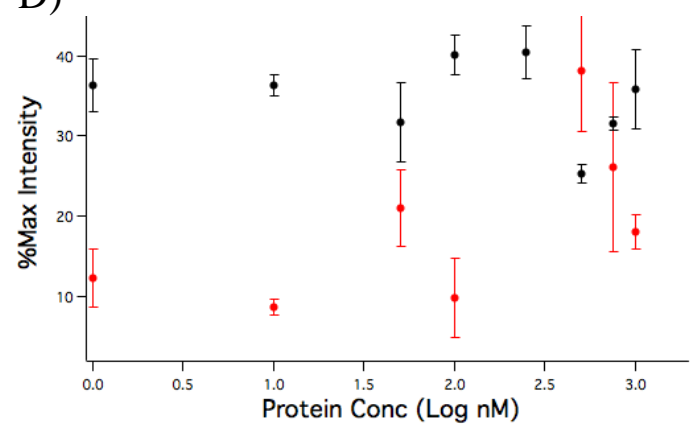


E)

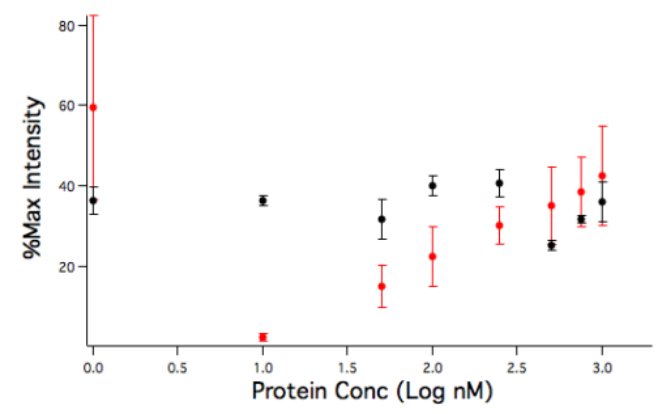

F)

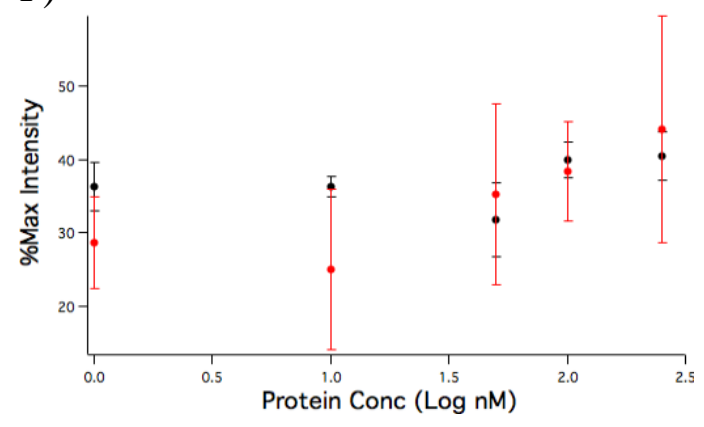

G)

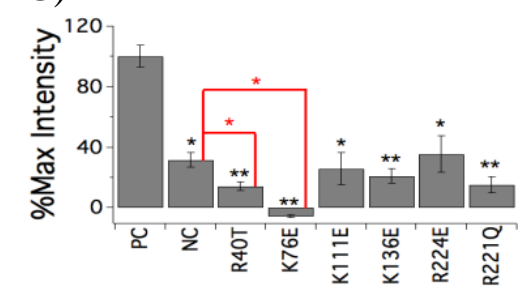

Figure 7. Mutations that show no dependence on protein concentration. The data represents $n=5$ samples and was analyzed using standard deviation (error bars). The negative control (•) samples were compared against the following mutations (•):A) R40T, B) K76E, C) K111E, D) K136E, E) R221Q, and F) R224E. G) Analysis of the statistical significance of each mutant at 50nM relative to the positive control where * denotes $\mathrm{p}<0.05,{ }^{* *}$ denotes $\mathrm{p}<0.005$; and the statistical significance of each measurement relative to the negative control where $*$ denotes $\mathrm{p}<0.05$.

ACCH lipid reorganization. After identification of mutations that cause a significant or total loss of the ACCH domain vesicle fusion activity, we endeavored to determine the relationship between membrane fusion and lipid organization. Hence, we incubated the lipid mixture with the K49E ACCH domain mutant that showed reduced fusion activity and used SAXS to characterize the lipid phases present in the mixture. $20 \mathrm{mM}$ of POPC/POPE/PI multi-lamellar lipid mixture was mixed with 0-0.07 mM protein resulting in the SAXS pattern shown in Figure 8A. Figure 8C shows that the K49E mutant does not merge the two dominant peaks in the range of 0.0112-0.123 $\AA$-1 as seen with the wild type protein in Figure 8B.

As previously discussed, this system contained four dominant Bragg peaks at $\sim 0.104$, $0.116,0.119$, and $0.122 \AA$-1. The wild type ACCH domain was shown to cause the 0.119 and 0.122 
$\AA$ A -1 to form a merged peak at $\sim 0.122 \AA$-1as the concentration of the protein increased (Figure $8 \mathrm{~B}$ ). Instead of merging the peaks, the K49E mutant appears to retain 4 peaks regardless of the protein concentration (Figure 8C). In fact, the only change between the lipid mixture pre- and post- protein incubation is that these peaks shifted to the lower q-values at a rate $\sim 0.041 \AA-1 / \mathrm{mM}$ protein (Figure 8D). This shift in q-values result suggests that the K49E mutant does associate with the lipid causing the d-spacing to increase to accommodate the protein. However, the retention of the dominate peaks suggests a loss of lipid phase reorganization activity. As this mutation had decreased vesicle fusion activity, this result demonstrates that the ACCH domain lipid reorganization activity and membrane fusion activity have some sort of inter-dependency.

A)

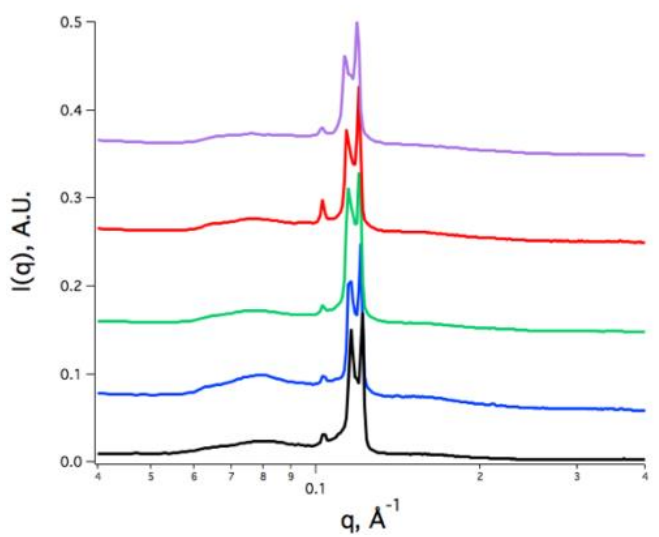

B)

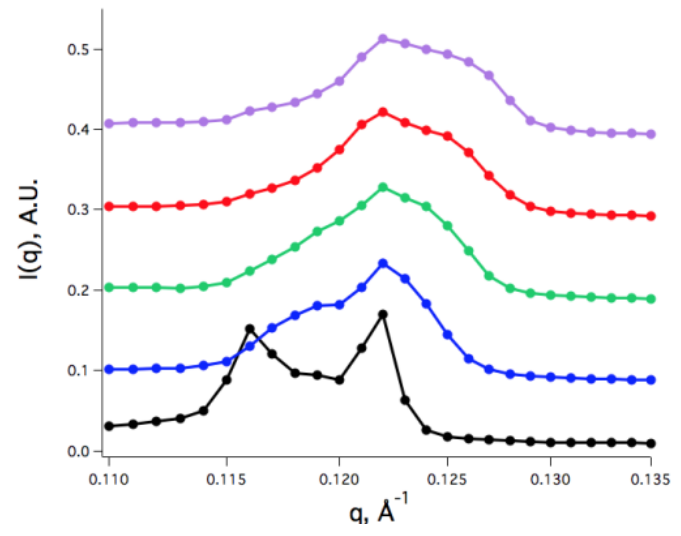

D)

C)

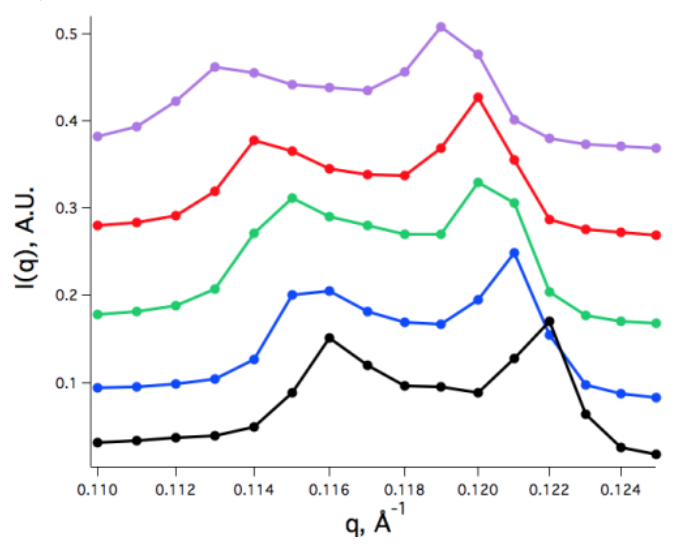




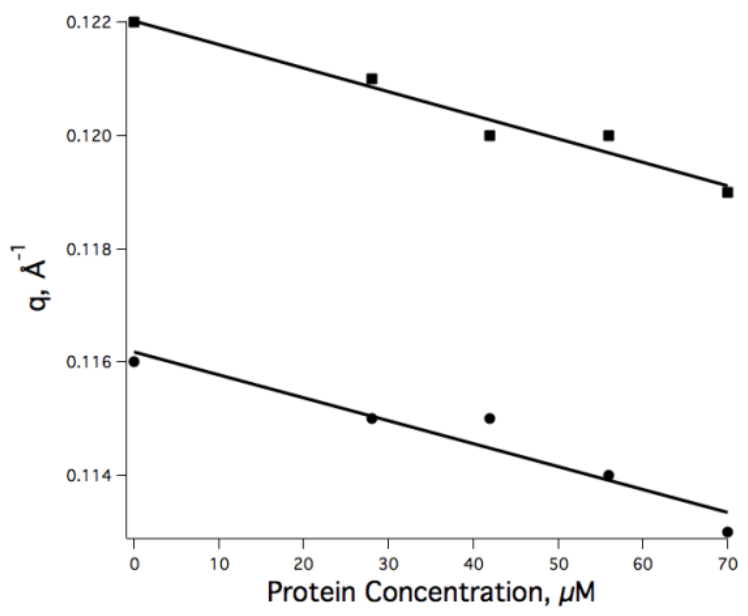

Figure 8. Mutating Lys49 Prevents ACCH Lipid Reorganization. A) Small angle xray scattering patterns of 20mM POPC/POPE/PI 60/20/20 mol\% incubated with 0 , $0.028,0.042,0.056$, and $0.07 \mathrm{mM} \mathrm{K49E}$ protein (-, -, -, -, - respectively). Highlighted Bragg peaks that are B) merged by wt protein are compared with C) the non-merged peaks seen when incubated with K49E. D) The two prominent peaks that merge for wt become shift to lower q-values (more hydrated) as a function of protein concentration. The peaks positions are fitted to 2 lines, were the slopes are $0.042 \pm 0.005\left(\mathrm{R}_{2}=0.96\right)$ and $0.041 \pm 0.007\left(\mathrm{R}_{2}=0.92\right) \AA-1 / \mathrm{mM}$ protein.

Based on the swelling trends seen beyond the concentration of the vesicle fusion FRET assay for this mutation, we would suggest that all of class II mutations (significantly reduced fusion activity) and class III mutations (complete loss of fusion activity) would lead to a loss of lipid phase reorganization at these concentrations. This is interesting as the trend of the class II mutants would suggest that K49E protein concentrations higher than $0.001 \mathrm{mM}$ should lead to the some vesicle fusion occurring. Instead, the SAXS data suggested that any fusion that occurred was not great enough to cause lipid reorganization to occur. Hence, we hypothesized that fusion activity is necessary for lipid reorganization to occur. Future experimentation with unilamellar vesicles to address globular changes in dimension of the vesicles with both wild type and class II and class III mutations similar to the work done by Heller et al (2010) would further untangle whether the reorganization was arrested before or during the act of vesicle fusion.[13] This would allow for a 
more thorough explanation of the mechanism by which Amot80/130 association with golgi apparatus vesicles targets polarity proteins to the plasma membrane.

\section{Materials and Methods}

Materials. Fluorescent dyes, Fluor® 430 streptavidin and Alexa Fluor® 594 streptavidin were purchased from Fisher Scientific (Pittsburgh, PA). 1-Palmitoyl-2-oleoyl-sn-glycero-3phosphocholine (POPC), 1-palmitoyl-2-oleoyl-sn-glycero-3-phosphoethanolamine (POPE), and Soy L- $\alpha$-phosphatidylinositol (PI) were from Avanti Polar Lipids (Alabaster, AL). Dodecyl thiomaltopyranoside was obtained from Anatrace (Maumee, $\mathrm{OH}$ ). All other materials were purchased from Fisher Scientific (Pittsburgh, PA). Unless otherwise noted, the buffer solution used for all experiments contained $50 \mathrm{mM}$ Tris, $600 \mathrm{mM}$ HEPES, $300 \mathrm{mM} \mathrm{NaCl}, 0.5 \mathrm{mM}$ EDTA, 1 mM DTT, 4 mM Benzamidine, and 24.7 $\mu \mathrm{M}$ dodecyl thiomaltopyranoside.

Protein Purification. The Amot ACCH domain cDNA (UniProt ID Q4VCS5-2) and related GENEWIZ mutations (South Plainfield, NJ) were subcloned into the pGEX expression plasmid, transformed into Escherichia coli BL21 (DE3) cells, and purified as previously described.[16, 3140] In short, the GST-tagged protein was purified from cells pellets that were solubilized in lysis buffer (phosphate buffered saline solution containing $1 \mathrm{mM}$ DTT, $4 \mathrm{mM}$ Benzamidine, and 24.7 $\mu \mathrm{M}$ dodecyl thiomaltopyranoside), lysed using 50g/L lysozyme, and the lysates were then collected by centrifugation. Proteins were purified with glutathione resin by affinity chromatography batch purification[38-40] and eluted with an additional $50 \mathrm{mM}$ glutathione. The proteins were analyzed for purity using SDS-PAGE, and concentrated using a $10 \mathrm{kDa}$ filter tube to $\geq 32 \mu \mathrm{M}$.

Liposome Preparation. Liposomes were prepared following previously described lipid rehydration and equilibration methods, $[16,41,42]$ including heating above their melting 
temperatures to $90^{\circ} \mathrm{C}$ for an hour, 3-freeze/thaw cycles, sonicated for over an hour on an ice water bath and then stored at $4{ }^{\circ} \mathrm{C}$.

Fluorescence Resonance Energy Transfer (FRET). We have modified previously reported methods for determining vesicle fusion using FRET.[43, 44] In short, $5 \mu \mathrm{M}$ protein was incubated with liposomes containing $5 \mathrm{~mol} \%$ Biotin DHPE at the respective concentrations and rehydrated in $0.5 \mathrm{mg} / \mathrm{mL}$ of either Streptavidin labeled Alexa Fluor ${ }^{\circledR} 430$ or 594 . Positive and negative lipid mixture controls were determined based on Heller et al 2010 where normal lipid affinities were seen for 3/1/1 molar ratio POPC/POPE/PI liposomes and there was no affinity for 3/1/1 molar ratio POPC/POPE/POPA. Mutation screens were performed against 20mM lipid (POPC/POPE/PI 3/1/1 molar ratio). Triplicate measurements were determined from $434 \mathrm{~nm}$ excitation/617nm emission using the Molecular Devices Flexstation II384 (SoftMax ${ }^{\circledR}$ Pro v5.3). FRET intensity of the dyes (F) with respect to each protein concentration are reported as \%Max Intensity using the following equation:

$$
\% \text { Max Intensity }=\left(\mathrm{F}_{\mathrm{n}} / \mathrm{F}_{\max }\right) * 100 \quad \text { Equation } 1
$$

\%Max Intensity versus protein concentration was then fit to a straight line (SigmaPlot version 10.0, StatSys) to determine the burst slope $(0-200 \mathrm{nM})$.

Small-Angle X-ray Scattering. Measurements were performed using Advanced Photon Source (APS/ANL) beamline 12-ID-B. The pinhole and detector setup have been previously described elsewhere.[45] Samples were analyzed using a photon energy of $14 \mathrm{keV}$ within a capillary where sample loading was automated using a microfluidics machine that induced shear stress on the sample. Due to the induced shear stress, peaks may have shifted from previous publications. 
2D scattering data for five shots were averaged and integrated over the chi angle to obtain intensity versus $q\left(\AA_{-1}\right)$. The Lorentzian function, which fits intensity scattering curves using script in Igor version 6.3, was used to determine the peak position from the scattering curves.[16, 17] Resulting peak positions (q) were fitted for the presence of repeating unit lamellar structures, also known as the d-spacing (d), that is calculated from using Equation 2.

$$
\mathrm{q} 0,0, \mathrm{n}=2 \pi / \mathrm{d} \quad \text { Equation } 2
$$

\section{AUTHOR INFORMATION}

\section{Corresponding Author}

Corresponding Author: Ann C. Kimble-Hill

Corresponding Author Information: (317) 278-1763. ankimble@iu.edu

\section{Author Contributions}

All authors have given approval to the final version of the manuscript. A.K.-H. designed study, data analysis and manuscript preparation; S.S. performed FRET experiments, data analysis, and manuscript preparation; B.E. performed SAXS data analysis and manuscript preparation.

\section{Funding Sources}

This work was supported, in whole or in part, by National Institutes of Health/National Cancer Institute Grant K01-CA169078 and the IUPUI Center for Research and Learning Undergraduate Research Opportunities Program (UROP).

\section{Acknowledgments}


The authors thank Dr. Quyen Hoang who provided the FlexStation II used for this study. Special thanks to Dr. Clark D. Wells who provided the initial Amot constructs. SAXS Results shown in this work were performed at Argonne National Laboratory, X-ray Science Division at the Advanced Photon Source with the assistance of Beamline Staff member Xiaobing Zuo. Argonne is operated by UChicago Argonne, LLC, for the U.S. Department of Energy, Office of Biological and Environmental Research under contract DE-AC02-06CH11357.

\section{REFERENCES}

1. Wells, C. D.; Fawcett, J. P.; Traweger, A.; Yamanaka, Y.; Goudreault1, M.; Elder, K.; Kulkarni, S.; Gish, G.; Virag, C.; Lim, C.; Colwill, K.; Starostine, A.; Metalnikov, P.; Pawson, T., A Rich1/Amot Complex Regulates the Cdc42 GTPase and Apical-Polarity Proteins in Epithelial Cells. Cell 2006, 125, (3), 535-548

2. Vogt, P.; Hart, J.; Gymnopoulos, M.; Jiang, H.; Kang, S.; Bader, A.; Zhao, L.; Denley, A., Phosphatidylinositol 3-Kinase: The Oncoprotein. In Phosphoinositide 3-kinase in Health and Disease, Rommel, C.; Vanhaesebroeck, B.; Vogt, P. K., Eds. Springer Berlin Heidelberg: 2011; Vol. 347, pp 79-104.

3. Ranahan, W. P.; Han, Z.; Smith-Kinnaman, W.; Nabinger, S. C.; Heller, B.; Herbert, B.S.; Chan, R.; Wells, C. D., The Adaptor Protein AMOT Promotes the Proliferation of Mammary Epithelial Cells via the Prolonged Activation of the Extracellular SignalRegulated Kinases. Cancer Research 2011, 71, (6), 2203-2211.

4. Zhao, B.; Lei, Q.-Y.; Guan, K.-L., The Hippo-YAP pathway: new connections between regulation of organ size and cancer. Current Opinion in Cell Biology 2008, 20, (6), 638646.

5. Mauviel, A.; Nallet-Staub, F.; Varelas, X., Integrating developmental signals: a Hippo in the (path)way. Oncogene 2012, 31, (14), 1743-56.

6. Bao, Y.; Hata, Y.; Ikeda, M.; Withanage, K., Mammalian Hippo pathway: from development to cancer and beyond. Journal of biochemistry 2011, 149, (4), 361-79.

7. Adler, J. J.; Heller, B. L.; Bringman, L. R.; Ranahan, W. P.; Cocklin, R. R.; Goebl, M. G.; Oh, M.; Lim, H. S.; Ingham, R. J.; Wells, C. D., Amot130 Adapts Atrophin-1 Interacting Protein 4 to Inhibit Yes-associated Protein Signaling and Cell Growth. J Biol Chem 2013, 288, (21), 15181-93.

8. Zhao, B.; Li, L.; Lu, Q.; Wang, L. H.; Liu, C. Y.; Lei, Q.; Guan, K. L., Angiomotin is a novel Hippo pathway component that inhibits YAP oncoprotein. Genes Dev 2011, 25, (1), 51-63.

9. Chan, S. W.; Lim, C. J.; Chong, Y. F.; Pobbati, A. V.; Huang, C.; Hong, W., Hippo Pathway-independent Restriction of TAZ and YAP by Angiomotin. The Journal of Biological Chemistry 2011, 286, (9), 7018-7026.

10. Bratt, A.; Birot, O.; Sinha, I.; Veitonmäki, N.; Aase, K.; Ernkvist, M.; Holmgren, L., Angiomotin Regulates Endothelial Cell-Cell Junctions and Cell Motility. Journal of Biological Chemistry 2005, 280, (41), 34859-34869. 
11. Ernkvist, M.; Birot, O.; Sinha, I.; Veitonmaki, N.; Nystrom, S.; Aase, K.; Holmgren, L., Differential roles of p80- and p130-angiomotin in the switch between migration and stabilization of endothelial cells. Biochim Biophys Acta 2008, 1783, (3), 429-37.

12. Adler, J. J.; Johnson, D. E.; Heller, B. L.; Bringman, L. R.; Ranahan, W. P.; Conwell, M. D.; Sun, Y.; Hudmon, A.; Wells, C. D., Serum deprivation inhibits the transcriptional coactivator YAP and cell growth via phosphorylation of the 130-kDa isoform of Angiomotin by the LATS1/2 protein kinases. Proceedings of the National Academy of Sciences 2013.

13. Heller, B.; Adu-Gyamfi, E.; Smith-Kinnaman, W.; Babbey, C.; Vora, M.; Xue, Y.; Bittman, R.; Stahelin, R. V.; Wells, C. D., Amot Recognizes a Juxtanuclear Endocytic Recycling Compartment via a Novel Lipid Binding Domain. Journal of Biological Chemistry 2010, 285, (16), 12308-12320.

14. Chen, Z. L.; Zhao, X. H.; Wang, J. W.; Li, B. Z.; Wang, Z.; Sun, J.; Tan, F. W.; Ding, D. P.; Xu, X. H.; Zhou, F.; Tan, X. G.; Hang, J.; Shi, S. S.; Feng, X. L.; He, J., microRNA92a promotes lymph node metastasis of human esophageal squamous cell carcinoma via E-cadherin. J Biol Chem 2011, 286, (12), 10725-34.

15. Peck, C. J.; Virtanen, P.; Johnson, D. E.; Kimble-Hill, A. C., Using the predicted structure of the Amot Coiled Coil Homology domain to understand lipid binding. Indiana University Journal of Undergradute Research 2018, (In Press).

16. Kimble-Hill, A. C.; Petrache, H. I.; Seifert, S.; Firestone, M. A., Reorganization of Ternary Lipid Mixtures of Non-Phosphorylated Phosphatidylinositol Interacting with Angiomotin. The Journal of Physical Chemistry B 2018.

17. Johnson, M. A.; Seifert, S.; Petrache, H. I.; Kimble-Hill, A. C., Phase Coexistence in Single-Lipid Membranes Induced by Buffering Agents. Langmuir 2014, 30, (33), 98809885.

18. Hall, L. C.; Donovan, E.; Araya, M.; Idowa, E.; Jiminez-Segovia, I.; Folck, A.; Wells, C. D.; Kimble-Hill, A. C., Identification of Specific Lysines and Arginines That Mediate Angiomotin Membrane Association. ACS Omega 2019, 4, (4), 6726-6736.

19. Burkhard, P.; Stetefeld, J.; Strelkov, S. V., Coiled coils: a highly versatile protein folding motif. Trends in Cell Biology 2001, 11, (2), 82-88.

20. Gallop, J. L.; Jao, C. C.; Kent, H. M.; Butler, P. J. G.; Evans, P. R.; Langen, R.; McMahon, H. T., Mechanism of endophilin N-BAR domain-mediated membrane curvature. The EMBO Journal 2006, 25, (12), 2898-2910.

21. Gallop, J. L.; McMahon, H. T., BAR domains and membrane curvature: bringing your curves to the BAR. Biochem Soc Symp 2005, (72), 223-231.

22. Lin, J.-r.; Hu, J., SeqNLS: Nuclear Localization Signal Prediction Based on Frequent Pattern Mining and Linear Motif Scoring. PLoS One 2013, 8, (10), e76864.

23. Yi, C.; Shen, Z.; Stemmer-Rachamimov, A.; Dawany, N.; Troutman, S.; Showe, L. C.; Liu, Q.; Shimono, A.; Sudol, M.; Holmgren, L.; Stanger, B. Z.; Kissil, J. L., The p130 Isoform of Angiomotin Is Required for Yap-Mediated Hepatic Epithelial Cell Proliferation and Tumorigenesis. Science signaling 2013, 6, (291), ra77-ra77.

24. Neurath, H., The Role of Glycine in Protein Structure. Journal of the American Chemical Society 1943, 65, (10), 2039-2041.

25. McNew, J. A.; Weber, T.; Engelman, D. M.; Sollner, T. H.; Rothman, J. E., The length of the flexible SNAREpin juxtamembrane region is a critical determinant of SNAREdependent fusion. Mol Cell 1999, 4, (3), 415-21. 
26. Tokumaru, H.; Umayahara, K.; Pellegrini, L. L.; Ishizuka, T.; Saisu, H.; Betz, H.; Augustine, G. J.; Abe, T., SNARE Complex Oligomerization by Synaphin/Complexin Is Essential for Synaptic Vesicle Exocytosis. Cell 2001, 104, (3), 421-432.

27. Scales, S. J.; Chen, Y. A.; Yoo, B. Y.; Patel, S. M.; Doung, Y.-C.; Scheller, R. H., SNAREs Contribute to the Specificity of Membrane Fusion. Neuron 2000, 26, (2), $457-$ 464.

28. Deak, F.; Shin, O. H.; Kavalali, E. T.; Sudhof, T. C., Structural determinants of synaptobrevin 2 function in synaptic vesicle fusion. J Neurosci 2006, 26, (25), 6668-76.

29. Singh, A.; Hitchcock-DeGregori, S. E., Local Destabilization of the Tropomyosin Coiled Coil Gives the Molecular Flexibility Required for Actin Binding. Biochemistry 2003, 42, (48), 14114-14121.

30. Sudhof, T. C.; Rothman, J. E., Membrane fusion: grappling with SNARE and SM proteins. Science 2009, 323, (5913), 474-7.

31. Cha, R.; Tilly, W., PCR primer. Cold Spring Harbor Laboratory, Cold Spring Harbor, NY: 1995.

32. Flaman, J.-M.; Frebourg, T.; Moreau, V.; Charbonnier, F.; Martin, C.; Ishioka, C.; Friend, S. H.; Iggo, R., A rapid PCR fidelity assay. Nucleic acids research 1994, 22, (15), 3259.

33. Lundberg, K. S.; Shoemaker, D. D.; Adams, M. W. W.; Short, J. M.; Sorge, J. A.; Mathur, E. J., High-fidelity amplification using a thermostable DNA polymerase isolated from Pyrococcus furiosus. Gene 1991, 108, (1), 1-6.

34. Shugar, D., The measurement of lysozyme activity and the ultra-violet inactivation of lysozyme. Biochimica et Biophysica Acta 1952, 8, (0), 302-309.

35. Kimble-Hill, A. C.; Parajuli, B.; Chen, C.-H.; Mochly-Rosen, D.; Hurley, T. D., Development of Selective Inhibitors for Aldehyde Dehydrogenases Based on Substituted Indole-2,3-diones. Journal of Medicinal Chemistry 2014, 57, (3), 714-722.

36. Khanna, M.; Chen, C.-H.; Kimble-Hill, A.; Parajuli, B.; Perez-Miller, S.; Baskaran, S.; Kim, J.; Dria, K.; Vasiliou, V.; Mochly-Rosen, D.; Hurley, T. D., Discovery of a novel class of covalent inhibitor for aldehyde dehydrogenases. Journal of Biological Chemistry 2011.

37. Parajuli, B.; Kimble-Hill, A. C.; Khanna, M.; Ivanova, Y.; Meroueh, S.; Hurley, T. D., Discovery of novel regulators of aldehyde dehydrogenase isoenzymes. ChemicoBiological Interactions 2011, 191, (1-3), 153-158.

38. Petrosyan, A.; Ali, M. F.; Verma, S. K.; Cheng, H.; Cheng, P.-W., Non-muscle myosin IIA transports a Golgi glycosyltransferase to the endoplasmic reticulum by binding to its cytoplasmic tail. The International Journal of Biochemistry \& Cell Biology 2012, 44, (7), 1153-1165.

39. Ali, M. F.; Chachadi, V. B.; Petrosyan, A.; Cheng, P.-W., Golgi Phosphoprotein 3 Determines Cell Binding Properties under Dynamic Flow by Controlling Golgi Localization of Core $2 \mathrm{~N}$-Acetylglucosaminyltransferase 1. Journal of Biological Chemistry 2012, 287, (47), 39564-39577.

40. Bobba, S.; Ponnaluri, V. K. C.; Mukherji, M.; Gutheil, W. G., Microtiter Plate-Based Assay for Inhibitors of Penicillin-Binding Protein 2a from Methicillin-Resistant Staphylococcus aureus. Antimicrobial Agents and Chemotherapy 2011, 55, (6), 27832787. 
41. Koerner, Megan M.; Palacio, Luis A.; Wright, Johnnie W.; Schweitzer, Kelly S.; Ray, Bruce D.; Petrache, Horia I., Electrodynamics of Lipid Membrane Interactions in the Presence of Zwitterionic Buffers. Biophysical Journal 2011, 101, (2), 362-369.

42. Yamashita, Y.; Oka, M.; Tanaka, T.; Yamazaki, M., A new method for the preparation of giant liposomes in high salt concentrations and growth of protein microcrystals in them. Biochimica et Biophysica Acta (BBA) - Biomembranes 2002, 1561, (2), 129-134.

43. Gong, B.; Choi, B.-K.; Kim, J.-Y.; Shetty, D.; Ko, Y. H.; Selvapalam, N.; Lee, N. K.; Kim, K., High Affinity Host-Guest FRET Pair for Single-Vesicle Content-Mixing Assay: Observation of Flickering Fusion Events. Journal of the American Chemical Society 2015, 137, (28), 8908-8911.

44. Diao, J.; Ishitsuka, Y.; Lee, H.; Joo, C.; Su, Z.; Syed, S.; Shin, Y.-K.; Yoon, T.-Y.; Ha, T., A single vesicle-vesicle fusion assay for in vitro studies of SNAREs and accessory proteins. Nat Protoc 2012, 7, (5), 921-934.

45. Schmidt, O.; DeBartolo, J.; Kurtz, C.; Lee, B.; Seifert, S.; Winans, R.; Zuo, X., Variable q-range $\mathrm{x}$-ray scattering chamber for chemical and materials science at the Advanced Photon Source. Journal of Physics: Conference Series 2014, 493, 012010. 